\title{
First Demonstration of Symmetric 40-Gb/s TWDM-PON with 100-km Passive Reach and 1024-Split using Direct Modulation and Direct Detection
}

\author{
Lilin $\mathrm{Yi}^{{ }^{*}}$, Zhengxuan $\mathrm{Li}^{1}$, Weisheng $\mathrm{Hu}^{1}$, Xuelin Yang ${ }^{1}$, Shilin Xiao ${ }^{1}$, Hao He ${ }^{1}$, Jun Han ${ }^{2}$, Mingjun Gong ${ }^{2}$, \\ Chengbin Shen ${ }^{3}$ \\ ${ }^{1}$ State Key Lab of Advanced Optical Communication Systems and Networks, Shanghai Jiao Tong University, Shanghai 200240, \\ China,*lilinyi@sjtu.edu.cn \\ ${ }^{2}$ Oclaro Communication Technologies (Shanghai) Co., Ltd., 200253, China \\ ${ }^{3}$ China Telecom Corporation, Ltd., Shanghai Research Institute, 200122, China
}

\begin{abstract}
We propose and demonstrate a symmetric 40-Gb/s TWDM-PON supporting 100-km passive reach, 1024 split by using cost-effective direct modulation and direct detection for both upstream and downstream directions, without any repeater and dispersion compensator. OCIS codes: (060.2330) Fiber optics communications; (060.1155) All-optical networks
\end{abstract}

\section{Introduction}

Time and wavelength division multiplexed-passive optical network (TWDM-PON) has been selected as the primary solution for next generation passive optical network stage-2 (NG-PON2) by Full Service Access Network (FSAN) group, and its standardization is in progress by ITU-T [1]. Huawei has demonstrated the world-first 40/10-Gb/s TWDM-PON prototype with 38-dB loss budget [2]. To meet the increasing upstream bandwidth requirement, we have demonstrated a symmetric 40-Gb/s TWDM-PON system with 39-dB loss budget [3].

For the long-term goal of PON systems, 100-km distribution range and 1024 users are desired to consolidate central offices and reduce the cost per user [4], where at least 50-dB loss budget is required at ideal case. Even though electrical or optical repeaters can improve the loss budget [4-6], truly passive reach is desired to keep the "passive" characteristic of PON system. External modulation combined with coherent detection has been considered as a good solution for high loss budget [7-8]. Symmetric 10G-PON with loss budget up to $53 \mathrm{~dB}$ [7] and $51 \mathrm{~dB}$ [8] (considering the FEC limit BER of $3.8 \times 10^{-3}$ ) have been achieved by using complex modulation format and coherent digital receiver in optical line terminal (OLT) and/or optical network unit (ONU). From the viewpoint of commercial applications of NG-PON2, direct modulation and direct detection (DM-DD) are more mature and costeffective compared with the coherent solution. However 50-dB loss budget is a big challenge for the DM-DD PON system.

In this paper, to our best knowledge, we demonstrate the first symmetric 40-Gb/s TWDM-PON with 100-km truly passive reach and 1024 split using cost-effective DM-DD in both OLT and ONUs. The strong nonlinearitytolerance of DM enables high launching power therefore high downstream (DS) loss budget. An Erbiumdoped/Raman amplifier (EDRA) is used as a preamplifier in OLT to improve the upstream (US) loss budget. A single delay interferometer (DI) is employed in the OLT as an optical spectral reshaping filter to suppress the frequency chirp of directly-modulated lasers (DMLs) for all 8 channels, mitigating the fiber dispersion up to $2100 \mathrm{ps} / \mathrm{nm} / \mathrm{km}$. Finally, 51.2-dB loss budget is achieved, which is limited by the output power of the booster amplifier. The loss budget is as high as $56 \mathrm{~dB}$ for single channel case, showing the powerful capability of DM-DD.

\section{Proposed TWDM-PON configuration}

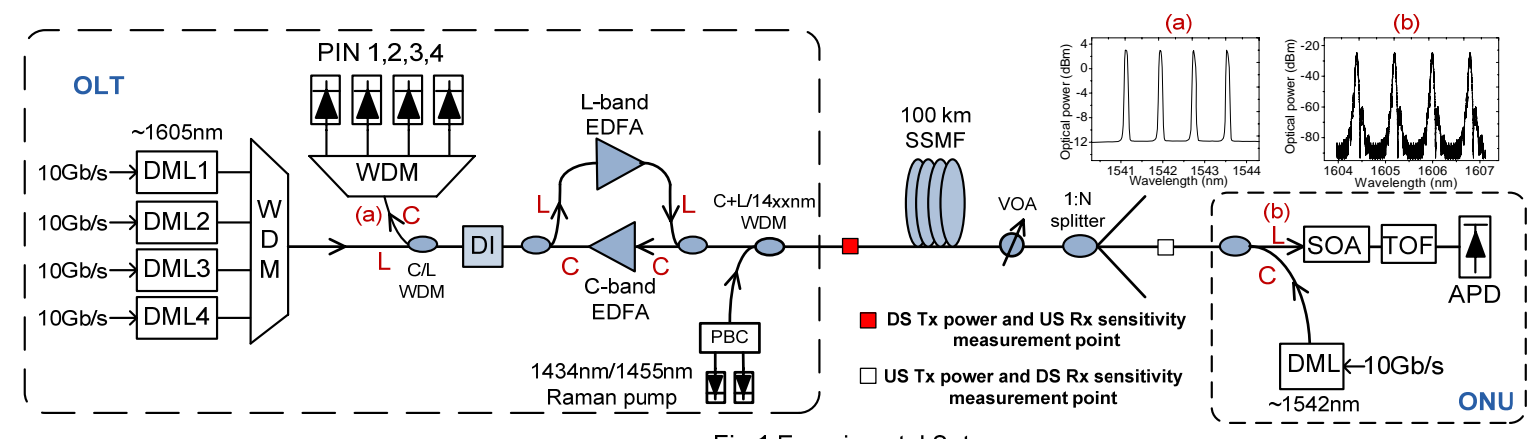

Fig.1 Experimental Setup 
Fig. 1 depicts the network configuration of the proposed repeater-less long reach TWDM-PON. In order to meet the twin constraints of low-cost and high performance for the access network, directly-modulated lasers (DMLs) are used as transmitters both in OLT and ONUs. The output wavelength of DML can be thermally tuned through 3 nm by varying its temperature, which is wide enough for TWDM-PON. Since the frequency chirp of DML induces strong dispersion in fiber link and limits the transmission distance of 10-Gb/s signal at tens of kilometers, a bandpass filter followed the DML, known as chirp-managed laser (CML), was proposed to mitigate the chirp-induced dispersion thereby extend the transmission distance [9]. In this work, a single DI is applied as a bi-pass and periodical notch filter to suppress the frequency chirp for all DS and US channels. For the DS direction, four DMLs combined with a DI act as a CML array but with lower cost. For the US direction, the 10-Gb/s directly-modulated signal transmitting through the long fiber is like a dispersion-supported transmission (DST) system [10], where the fiber dispersion firstly distorts the signal and then converts the frequency modulation into intensity modulation. The DI at the fiber end suppresses the residual frequency chirp therefore achieving clear eye opening even at the chirpdominated transmission distance. In this configuration, a single DI in OLT can manage the dispersion for all users; therefore no dispersion compensation is required, which is simple and cost-effective for commercial applications. We have proved this method was also applicable for burst-mode data [3]. Besides, the broad spectrum of the DM signal has stronger nonlinearity-tolerance than other modulation formats with carrier and the high power induced self-phase modulation (SPM) can benefit the receiver sensitivity [9], therefore a high power L-band erbium-doped fiber amplifier (EDFA) is allowed to use in OLT to improve the DS loss budget. A C-band EDRA in OLT is used to improve the receiver sensitivity for all upstream signals, which could achieve similar or even superior performance compared with coherent receivers but with much lower complexity. All the components used in this configuration are commercially available with mass production capability.

\section{Experimental setup and results}
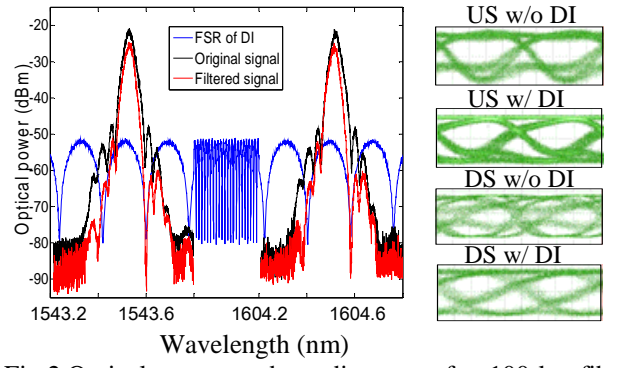

Tab.1 Loss budget evaluation
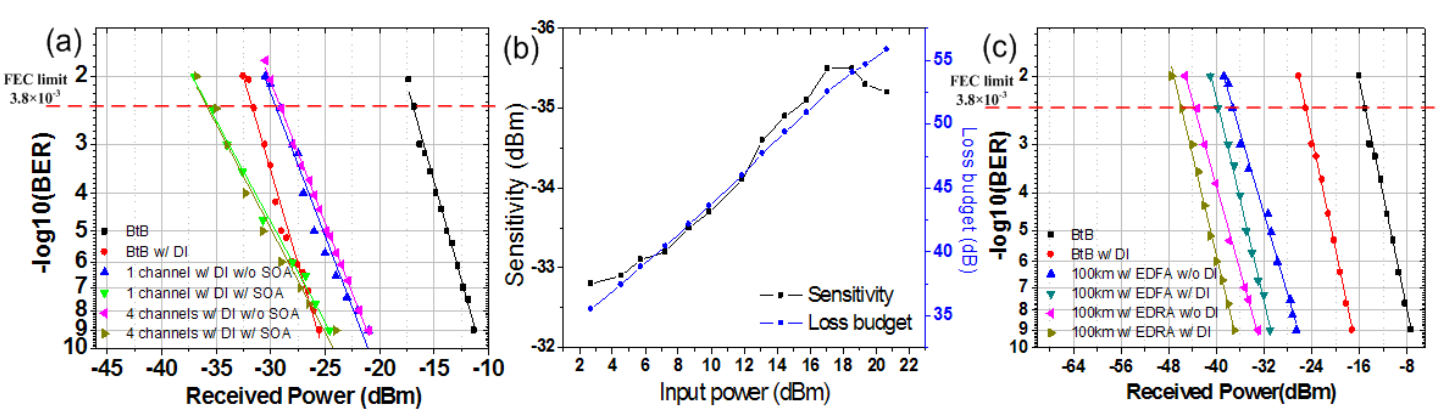

Fig.3 (a) BER measurement for DS signal (b) Sensitivity and loss budget variation for DS signal (c) BER measurement for US signal

An experiment was performed to investigate the performance of the proposed TWDM-PON. The DML had a maximal output power of $11 \mathrm{dBm}$. Four DMLs operating at $1605 \mathrm{~nm}$ and another four DMLs operating at $1542 \mathrm{~nm}$ under room temperature served as DS and US transmitters, respectively. The output wavelengths with 100-GHz channel spacing are shown in the insets of Fig.1. In the DS direction, the multiplexed channels firstly passed through the DI for chirp management and then boosted by the L-band EDFA, then launched into 100-km standard singlemode fiber (SSMF) with 20-dB loss and a chromatic dispersion of $2100 \mathrm{ps} / \mathrm{nm} / \mathrm{km}$ at $1605 \mathrm{~nm}$. The maximal launching power was $16 \mathrm{dBm} / \mathrm{ch}$ limited by the EDFA. A variable optical attenuator (VOA) before the ONU was used to imitate the splitting loss. In each ONU, an L-band semiconductor optical amplifier (SOA) with 18-dB gain and 7.5-dB noise figure (NF) at $1605 \mathrm{~nm}$ amplified all the four downstream signals and then an L-band tunable optical filter (TOF) selected the desired channel and suppressed the amplified spontaneous emission (ASE). A 10- 
GHz APD was used in each ONU to achieve high receiver sensitivity. For the US detection, the DI located right after the C-band EDRA can achieve the best receiver sensitivity. The Raman amplifier provided a 10-dB small signal gain at $1540 \mathrm{~nm}$ with two 250-mW pumps operating at $1434 \mathrm{~nm}$ and $1455 \mathrm{~nm}$. The EDFA was with 45-dB gain and 3.9-dB NF at $1540 \mathrm{~nm}$. A WDM demux followed by 10-GHz PINs were used to directly detect the desired US channel. For each DML, the modulated data were 10-Gb/s PRBS with a word length of231-1.

Fig. 2 shows the optical spectra and eye diagrams of the original and reshaped signals by DI for both US and DS directions after 100-km fiber transmission. By suppressing the long wavelength chirp corresponding to " 0 ” levels, the extinction ratio (ER) is improved and the eye becomes clearly open even after 100-km fiber transmission for both US and DS signals. Note that the eye of C-band US signal is open after 100-km fiber due to DST effect [9], but it doesn't work for L-band. The free-spectral-range (FSR) of DI is set at $25 \mathrm{GHz}$ for simultaneous multi-channel operation with 100-GHz channel spacing. In practical applications, the wavelengths of the DMLs need to align with DI by feedback control. From simulation, +/- $5 \mathrm{GHz}$ frequency drift affects ER by less than $1 \mathrm{~dB}$.

The bit error rate (BER) and sensitivity measurement for DS and US signals are shown in Fig.3. For both DS and US directions, all channels have almost same BER performance, therefore only 1604.3nm for DS and 1534.5nm for US are selected as example. For the DS direction, DI can improve the back-to-back (BtB) sensitivity (considering the FEC limit BER of $3.8 \times 10-3$ ) of DML from $-17.5 \mathrm{dBm}$ to $-31.5 \mathrm{dBm}$ due to ER improvement. After $100-\mathrm{km}$ fiber transmission, the power penalty is around $2.5 \mathrm{~dB}$, but an L-band SOA can improve the sensitivity by $6 \mathrm{~dB}$, so finally $-35.2-\mathrm{dBm}$ sensitivity can be achieved for both single channel and 4 channels cases. Fig 3(b) shows the sensitivity variation with the launching power of the DS single channel. The high power induced SPM benefits the sensitivity therefore the loss budget is improved with the input power increase. The highest loss budget for single channel is $55.9 \mathrm{~dB}$ at $20.7-\mathrm{dBm}$ launching power, which is the maximal power for single-channel input case. For 4 channels case, the maximal launching power is $16 \mathrm{dBm} / \mathrm{ch}$, corresponding to 51.2-dB loss budget. But even for the maximal 22-dBm launching power, the sensitivity degradation is not observed, so we believe the DS loss budget could be further improved by using an L-band EDFA with higher power. For the US direction, DI can improve the BtB sensitivity from $-15.5 \mathrm{dBm}$ to $-25.5 \mathrm{dBm}$. After transmitting 100-km fiber, even without DI, the sensitivity can achieve $-37 \mathrm{dBm}$ with EDFA only due to DST effect. By using EDRA as preamplifier, the sensitivity is improved by $7 \mathrm{~dB}$. The DI after the EDRA can further improve the sensitivity by $2 \mathrm{~dB}$. Finally $-46 \mathrm{dBm}$ sensitivity can be achieved, even better than the coherent receiver [7]. Considering the $10-\mathrm{dBm}$ launching power of DML, the US loss budget is 56 $\mathrm{dB}$. Table 1 shows the detailed US and DS loss budget, where the loss of all passive components except for fiber and splitter has already been excluded.

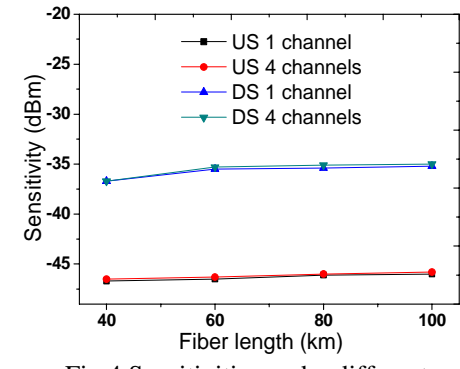

Fig.4 Sensitivities under different transmission distances

Finally, to verify that the proposed TWDM- PON is suitable for ONUs located at different transmission distances, the fiber length is varied from $40 \mathrm{~km}$ to $100 \mathrm{~km}$ with a step of $20 \mathrm{~km}$. From Fig. 4 we can see the sensitivities of both DS and US signals are almost independent with the transmission distances. The residual Raman pump power at ONU is less than $-20 \mathrm{dBm}$ at $40-\mathrm{km}$ distance, shows negligible effects on both US and DS performance.

\section{Conclusion}

We have proposed and demonstrated a long-reach symmetric 40-Gb/s TWDM-PON using DM-DD for both DS and US directions. A single DI reshapes the signal spectra of all channels for mitigating the dispersion. An L-band high power EDFA, an L-band SOA and a C-band EDRA are used to increase the loss budget to $51.2 \mathrm{~dB}$, which could support 1024 users within a truly-passive distribution range up to $100 \mathrm{~km}$ at ideal case, and can be further improved if L-band EDFA with higher power is available. The proposed architecture provides a cost-effective solution for long reach and large split symmetric 40-Gb/s TWDM-PON.

\section{Acknowledgements}

This work was supported by Nature Science Foundation China (61322507, 61132004) and Program of Excellent PhD in China (201155).

\section{References}

[1]ITU-T Recommendation G.989.2, (08/2013).

[2]Y.Ma et al., OFC'12, PDP5D.7 (2012).

[3]L.Yi et al., PTL vol. 25, p.644 (2013).

[4]D.P.Shea et al., JLT vol.25,p.685 (2007).

[5]T.V.Lerber et al., OE vol.19, p.B645(2011).

[6]P.P.Iannone et al., OFC'11,PDPD6 (2011).

[7]D.Lavery et al., OFC'11, OTuB4 (2011).

[8]D.Qian et al., ECOC'12, Tu.1.B.2 (2012).

[9]S.Chandarsekhar et al.,OFC'05,PDP30 (2005). [10]B.Wedding et al., JLT vol.12, p.1720 (1994). 